\title{
Evaluating named entity recognition tools for extracting social networks from novels
}

\author{
Niels Dekker ${ }^{1}$, Tobias Kuhn ${ }^{1}$, Marieke van Erp ${ }^{\text {Corresp. } 2}$ \\ ${ }^{1}$ Department of Computer Science, Vrije Universiteit Amsterdam, Amsterdam, The Netherlands \\ 2 DHLab, KNAW Humanities Cluster, Amsterdam, The Netherlands \\ Corresponding Author: Marieke van Erp \\ Email address: marieke.van.erp@dh.huc.knaw.nl
}

The analysis of literary works has experienced a surge in computer-assisted processing. To obtain insights into the community structures and social interactions portrayed in novels, the creation of social networks from novels has gained popularity. Many methods rely on identifying named entities and relations for the construction of these networks, but many of these tools are not specifically created for the literary domain. Furthermore, many of the studies on information extraction from literature typically focus on 19th and early 20th century source material. Because of this, it is unclear if these techniques are as suitable to modern-day literature as they are to those older novels. We present a study in which we evaluate natural language processing tools for the automatic extraction of social networks from novels as well as their network structure. We find that there are no significant differences between old and modern novels but that both are subject to a large amount of variance. Furthermore, we identify several issues that complicate named entity recognition in our set of novels and we present methods to remedy these. We see this work as a step in creating more culturally-aware Al systems. 


\title{
- Evaluating named entity recognition tools for extracting social networks from novels
}

Corresponding author:

Marieke van Erp ${ }^{2}$

Email address: marieke.van.erp@dh.huc.knaw.nl

\begin{abstract}
The analysis of literary works has experienced a surge in computer-assisted processing. To obtain insights into the community structures and social interactions portrayed in novels, the creation of social networks from novels has gained popularity. Many methods rely on identifying named entities and relations for the construction of these networks, but many of these tools are not specifically created for the literary domain. Furthermore, many of the studies on information extraction from literature typically focus on 19th and early 20th century source material. Because of this, it is unclear if these techniques are as suitable to modern-day literature as they are to those older novels. We present a study in which we evaluate natural language processing tools for the automatic extraction of social networks from novels as well as their network structure. We find that there are no significant differences between old and modern novels but that both are subject to a large amount of variance. Furthermore, we identify several issues that complicate named entity recognition in our set of novels and we present methods to remedy these. We see this work as a step in creating more culturally-aware Al systems.
\end{abstract}

\section{INTRODUCTION}

The characters and their relations can be seen as the backbone of any story, and explicitly creating and analysing a network from these relationships can provide insights into the community structures and social interactions portrayed in novels (Moretti, 2013). Quantitative approaches to social network analysis to examine the overall structure of these social ties, are borrowed from modern sociology and have found their way into many other research fields such as computer science, history and literary studies (Scott, 2012). Elson et al. (2010), Lee and Yeung (2012), Agarwal et al. (2013), and Ardanuy and Sporleder (2014) have all proposed methods for automatic social network extraction from literary sources. The most commonly used approach for extracting such networks, is to first identify characters in the novel through Named Entity Recognition (NER) and then identifying relationships between the characters through for example measuring how often two or more characters are mentioned in the same sentence or paragraph.

Many studies use off-the-shelf named entity recognisers, which are not necessarily optimised for the literary domain and do not take into account the surrounding cultural context. Furthermore, to the best of our knowledge, such studies focus on social network extraction from 19th and early 20th century novels (which we refer to as classic novels). ${ }^{1}$ Typically, these classic novels

\footnotetext{
${ }^{1}$ We follow (Sainte-Beuve, 1910) here in defining a classic novel not as one written by the ancient Greeks or Romans ('the classics') but to canonical works.
} 
are obtained from Project Gutenberg, ${ }^{2}$ where such public domain books are available for free. While beneficial for the accessibility and reproducibility of the studies in question, more recent novels may not imitate these classic novels with respect to structure or style. It is therefore possible that classic novels have social networks that have a structure that is very different from more recent literature. They might differ, for example, in their overall number of characters, in the typical number of social ties any given character has, in the presence or absence of densely connected clusters, or in how closely connected any two characters are on average. Moreover, changes along dimensions such as writing style, vocabulary, and sentence length could prove to be either beneficial or detrimental to the performance of natural language processing techniques. This may lead to different results even if the actual network structures remained the same. Vala et al. (2015) did compare 18th and 19th century novels on the number of characters that appear in the story, but found no significant difference between the two. Furthermore, an exploration of extracted networks can also be used to assess the quality of the extracted information and investigate the structure of the expression of social ties in a novel.

Thus far, we have not found any studies that explore how named entity recognition tools perform on a diverse corpus of fiction literature. In this study, we evaluate four different tools on a set of classic novels which have been used for network extraction and analyses in prior work, as well as more recent fiction literature (henceforth referred to as modern novels). We need such an evaluation to assess the robustness of these tools to variation in language over time (Biber and Finegan, 1989) and across literary genres. Comparing social networks extracted from corpora consisting of classic and modern novels may give us some insights into what characteristics of literary text may aid or hinder automatic social network extraction and provide indications of cultural change.

As previous work (e.g. Ardanuy and Sporleder (2014)) has included works from different genres, in this work we decided to focus on the fantasy/science fiction domain to smooth potential genre differences in our modern books. In our evaluation, we devote extra attention to the comparison between classic and modern fantasy/science fiction in our corpus.

We define the following research questions:

- To what extent are off-the-shelf named entity recognition tools suitable for identifying fictional characters in novels?

- Which differences or similarities can be discovered between social networks extracted for different novels?

To answer our first research question, we first evaluate four named entity recognisers on 20 classic and 20 modern fantasy/science fiction novels. In each of these novels, the first chapter is manually annotated with named entities and coreference relations. The named entity recognisers we evaluate are: 1) BookNLP (Bamman et al., 2014) ${ }^{3}$ which is specifically tailored to identify and cluster literary characters, and has been used to extract entities from a corpus of 15,099 English novels. At the time of writing, this tool was cited 80 times. 2) Stanford NER version 3.8.0 (Finkel et al., 2005), one of the most popular named entity recognisers in the NLP research community, cited 2,648 times at the time of writing. 3) Illinois Named Entity Tagger version 3.0.23 (Ratinov and Roth, 2009), a computationally efficient tagger that uses a combination of machine learning, gazetteers, ${ }^{4}$ and additional features extracted from unlabelled data. At the time of writing, the system was downloaded over 10,000 times. Our last system (4) is IXA-Pipe-NERC version 1.1.1 (Agerri and Rigau, 2016), a competitive classifier that employs

\footnotetext{
${ }^{2}$ http: //gutenberg.org/

${ }^{3}$ https : / / github.com/dbamman/book-nlp - commit: 81d7a31

${ }^{4} \mathrm{~A}$ gazetteer is a list of names
} 
unlabelled data via clustering and gazetteers that outperformed other state-of-the-art named entity recognition (NER) tools on their within and out-domain evaluations.

To answer the second research question, we use the recognised named entities to create a co-occurrence network for each novel. Network analysis measures are then employed to compare the extracted networks from the classic and modern novels to investigate whether the networks from the different sets of novels exhibit major differences.

The contributions of this paper are: (1) a comparison and an analysis of four named entity recognition on 20 classic and 20 modern novels; (2) a comparison and an analysis of social network analysis measures on networks automatically extracted from 20 classic and 20 modern novels; (3) experiments and recommendations for boosting performance on recognising entities in novels; and (4) an annotated gold standard dataset with entities and coreferences of 20 classic and 20 modern novels.

The remainder of this paper is organised as follows. We first discuss related work Section 2. Next, we describe our approach and methods in Section 3. We present our evaluation of four different named entity recognition systems on 20 classic and 20 modern novels in Section 4, followed by the creation and analysis of social networks in Section 5. We discuss issues that we encountered in the identification of fictional characters and showcase some methods to boost performance in Section 6. We conclude by suggesting directions for future work in Section 7.

The code for all experiments as well as annotated data can be found at https://github. com/Niels-Dekker/Out-with-the-Old-and-in-with-the-Novel.

\section{RELATED WORK}

As mentioned in Section 1, we have not found any other studies that compared the performances of social network extraction on classic and modern novels; or compared the structures of these networks. This section therefore focuses on the techniques used on classic literature. In first part of this section, we will describe how other studies extract and cluster characters. In the second part, we outline what different choices can be made for the creation of a network, and motivate our choices for this study.

\section{Named Entity Recognition}

The first and foremost challenge in creating a social network of literary characters is identifying the characters. Named Entity Recognition is often used to identify passages in text that identify things by a name. Furthermore, identified passages are often also classified into various categories such as person, location, and organisation. Typically, this approach is also used to identify miscellaneous numerical mentions such as dates, times, monetary values and percentages.

Elson et al. (2010), Ardanuy and Sporleder (2014), Bamman et al. (2014) and Vala et al. (2015) all use the Stanford NER tagger (Finkel et al., 2005) to identify characters in literary fiction. On a collection of Sherlock Holmes novels, these studies perform Named Entity Recognition tasks with $F_{1}$-scores between: .45 and .54 . Vala et al. (2015) propose that the main difficulty with this collection is the multitude of minor characters, a problem which we expect to be also present in our collections of classic and modern novels.

A big difference between the news domain (for which most language technology tools have been created) and the literary domain, is that names do not have to follow the same 'rules' as names in the real world. This topic is explored in the Namescape project by De Does et al. (2017). ${ }^{5}$ In this project, 1 million tokens taken from 550 Dutch novels were manually annotated. A distinction between first and last names was made in order to test whether different name parts are used with different effects. A named entity recogniser was trained specifically for this corpus

\footnotetext{
${ }^{5}$ http: //blog.namescape.nl/
} 
by Van Dalen-Oskam et al. (2014), obtaining an $F_{1}$ score of 0.936 for persons. The corpus contains fragments of novels written between the 17th and 20th century, but as the corpus and tools are not available, we cannot investigate its depth or compare it directly to our work. Other approaches attempt to use the identification of locations and physical proximity to improve the creation of a social network (Lee and Yeung, 2012).

\section{Coreference resolution}

One difficulty of character detection is the variety of aliases one character might go by, or; coreference resolution. For example, George Martin's Tyrion Lannister, might alternatively be mentioned as Ser Tyrion Lannister, Lord Tyrion, Tyrion, The Imp or The Halfman. In the vast majority of cases, it is desirable to collapse those character references into one character entity. However, in some cases, retaining some distinction between character references can be useful: we provide an example of this in Subsection 5.4.

Two distinct approaches attempt to address this difficulty, (1) omit parts of a multi-word name, or (2) compile a list of aliases. The former approach leaves out honorifics such as the Ser and Lord in the above example in order to cluster the names of one character. To automate this clustering step, some work has been done by Bamman et al. (2014) and Ardanuy and Sporleder (2014). While useful, the former approach alone provides no solace for the matching of the last two example aliases; where no part of the character's name is present. The latter approach thus suggests to manually compile a list of aliases for each character with the aid of external resources or annotators. This method is utilised by Elson et al. (2010) and Lee and Yeung (2012). In Van Dalen-Oskam et al. (2014), wikification (i.e. attempting to match recognised names to Wikipedia resources) is used. Obviously this is most useful for characters that are famous enough to have a Wikipedia page. The authors state in their error analysis Van Dalen-Oskam et al. (2014, Section 3.2) that titles that are most likely from the fantasy domain are most difficult to resolve, which already hints at some differences between names in different genres.

\section{Anaphora resolution}

To identify as many character references as possible, it is important to take into account that not all references to a character actually mention the character's name. In fact, Bamman et al. (2014) show that $74 \%$ of character references come in the form of a pronouns such as he, him, his, she, her and hers in a collection of 15,099 English novels. To capture these references, the anaphoric pronoun is typically matched to its antecedent by using the linear word distance between the two, and by matching the gender of anaphora to that of the antecedent. The linear word distance can be, for example, the number of words between the pronoun and the nearest characters. For unusual names, as often found in science fiction and fantasy, identification of the gender may be problematic.

\section{Network Creation}

For a social network of literary characters, characters are represented by the nodes, whereas the edges indicate to some interaction or relationship. While the definition of a character is uniformly accepted in the literature, the definition of an interaction varies per approach. In previous research, two main approaches can be identified to define such an edge. On the one hand, conversational networks are used in approaches by Chambers and Jurafsky (2008), Elson and McKeown (2010) and He et al. (2013). This approach focuses on the identification of speakers and listeners, and connecting each speaker and listener to the quoted piece of dialogue they utter or receive. On the other hand, co-occurrence networks (as used by Ardanuy and Sporleder (2014) and Fernandez et al. (2015)) are created by connecting characters if they occur in the same body of text. While conversational networks can provide a good view of who speaks directly to whom, Ardanuy and Sporleder (2014) argue that “...much of the interaction 
in novels is done off-dialogue through the description of the narrator or indirect interactions" (p. 34). What value to assign to the edges depends on the end-goal of the study. For example, Fernandez et al. (2015) assign a negative or positive sentiment score to the edges between each character-pair in order to ultimately predict the protagonist and antagonist of the text. Ardanuy and Sporleder (2014) used weighted edges to indicate how often two characters interact.

\section{Network Analysis}

Social network analysis draws upon network theory for its network analysis measures (Scott, 2012). The application of these measures to networks extracted from literature has been demonstrated insightful in assessing the relationships of characters in for example 'Alice in Wonderland' (Agarwal et al., 2012) and 'Beowulf', the 'Iliad' and 'Táin Bó Cuailnge' ('The Cattle Raid of Cooley', an Irish epic) (Mac Carron and Kenna, 2012). Network analysis can also play a role in authorship attribution e.g.(Amancio, 2015; Akimushkin et al., 2017) and characterising a novel (Elson et al., 2010).

\section{MATERIALS AND DATA PREPARATION}

For the study presented here, we are interested in the recognition and identification of persons mentioned in classic and modern novels for the construction of the social network of these fictitious characters. We use off-the-shelf state-of-the-art entity recognition tools in an automatic pipeline without manually created alias lists or similar techniques. For the network construction, we follow Ardanuy and Sporleder (2014) and apply their co-occurrence approach for the generation of the social network links with weighted edges that indicate how often two characters are mentioned together. We leave the consideration of negative weights and sentiments for future work. Before we will explain the details of the used entity recognition tools, how they compare for the given task, and how their results can be used to build and analyse the respective social networks, we explain first the details of our selected corpus, how we preprocessed the data, and how we collected the annotations for the evaluation.

\subsection{Corpus Selection}

Our dataset consists of 40 novels - 20 classic and 20 modern novels - the specifics of which are presented in Table A2 in the Appendix. Any selection of sources is bound to be unrepresentative in terms of some characteristics but we have attempted to balance breadth and depth in our dataset. Furthermore, we have based ourselves on selections made by other researchers for the classics and compilations by others for the modern books.

For the classic set, the selection was based on Guardian's Top 100 all-time classic novels. ${ }^{6}$ Wherever possible, we selected books that were (1) analysed in related work (as mentioned in Subsection 2) and (2) available through Project Gutenberg. ${ }^{7}$

For the modern set, the books were selected by reference to a list compiled by BestFantasyBooksCom. ${ }^{8}$ For our final selection of these novels, we deliberately made some adjustments to get a wider selection. That is, some of the books in this list are part of a series. If we were to include all the books of the upvoted series, our list would consist of only 4 different series. We therefore chose to include only the first book of each of such series. As the newer books are unavailable on Gutenberg, these were purchased online. These digital texts are generally provided in .epub or .mobi format. In order to reliably convert these files into plain text format,

\footnotetext{
${ }^{6}$ The Guardian: https://www.theguardian.com/books/2003/oct/12/features.fiction Last retrieved: 30 October 2017

${ }^{7}$ https: / / www. gutenberg.org/

8 bestfantasybooks.com/top25-fantasy-books . php Last retrieved: 30 October 2017
} 
we used Calibre ${ }^{9}$ - a free and open-source e-book conversion tool. This conversion was mostly without any hurdles, but some issues were encountered in terms of encoding, as is discussed in the next section. Due to copyright restrictions, we cannot share this full dataset but our gold standard annotations of the first chapter of each are provided on this project's Github page. The ISBN numbers of the editions used in our study can be found in Table A2 the Appendix.

\subsection{Data Preprocessing}

To ensure that all the harvested text files were ready for processing, we firstly ensured that the encoding for all the documents was the same, in order to avoid issues down the line. In addition, all information that is not directly relevant to the story of the novel was stripped. Even while peripheral information in some books - such as appendices or glossaries - can provide useful information about character relationships, we decided to focus on the story content and thus discard this information. Where applicable, the following peripheral information was manually removed: (1) reviews by fellow writers, (2) dedications or acknowledgements, (3) publishing information, (4) table of contents, (5) chapter headings and page numbers, and (6) appendices and/or glossaries.

During this clean-up phase, we encountered some encoding issues that came with the conversion to plain text files. Especially in the modern novels, some novels used inconsistent or odd quotation marks. This issue was addressed by replacing the inconsistent quotation marks with neutral quotations that are identical in form, regardless of whether if it is used as opening or closing quotation mark.

\subsection{Annotation}

Because of limitations in time and scope, we only annotated approximately 1 chapter of each novel. In this subsection, we describe the annotation process.

\section{Annotation Data}

To evaluate the performance for each novel, a gold standard was created manually. Two annotators (not the authors of this article) were asked to evaluate 10 books from each category. For each document, approximately one chapter was annotated with entity co-occurrences. Because the length of the first chapter fluctuated between 84 and 1,442 sentences, we selected an average of 300 sentences for each book that was close to a chapter-boundary. For example, for Alice in Wonderland, the third chapter ended on the 315th sentence, so the first three chapters were extracted for annotation. While not perfect, we attempted to strike a balance between comparable annotation lengths for each book, without cutting off mid-chapter.

\begin{tabular}{c|l|l|l|l|l|l}
\hline id & $\begin{array}{l}\text { Preceding } \\
\text { context }\end{array}$ & Focus sentence & $\begin{array}{l}\text { Subsequent } \\
\text { context }\end{array}$ & $\#$ & Person 1 & Person 2 \\
\hline 541 & $\begin{array}{l}\text { Bran reached } \\
\text { out hesitantly. }\end{array}$ & $\begin{array}{l}\text { "Go on,” Robb } \\
\text { told him. }\end{array}$ & $\begin{array}{l}\text { "You can touch } \\
\text { him." }\end{array}$ & 2 & $\begin{array}{l}\text { Robb } \\
\text { Stark }\end{array}$ & $\begin{array}{l}\text { Bran } \\
\text { Stark }\end{array}$ \\
\hline
\end{tabular}

Table 1. Annotation Example.

\section{Annotation Instructions}

For each document, the annotators were asked to annotate each sentence for the occurrence of characters. That is, for each sentence, identify all the characters in it. To describe this process, an example containing a single sentence from A Game of Thrones is included in Table 1. The id of

\footnotetext{
${ }^{9}$ https: / / calibre-ebook.com/ - version 2.78
} 
the sentence is later used to match the annotated sentence to its system-generated counterpart for performance evaluation. The focus sentence is the sentence that corresponds to this id, and is the sentence for which the annotator is supposed to identify all characters. As context, the annotators are provided with the preceding and subsequent sentences. In this example, the contextual sentences could be used to resolve the ' $\mathrm{him}$ ' in the focus sentence to 'Bran'. To indicate how many persons are present, the annotators were asked to fill in the corresponding number (\#) of people - with a maximum of 10 characters per sentence. Depending on this number of people identified, subsequent fields became available to the annotator to fill in the character names.

To speed up the annotation, an initial list of characters was created by applying the BookNLP pipeline to each novel. The annotators were instructed to map the characters in the text to the provided list to the best of their ability. If the annotator assessed that a person appears in a sentence, but is unsure of this character's identity, the annotators would mark this character as default. In addition, the annotators were encouraged to add characters, should they be certain that this character does not appear in the pre-compiled list, but occurs in the text nonetheless. Such characters were given a specific tag to ensure that we could retrieve them later for analysis. Lastly, if the annotator is under the impression that two characters in the list refer to the same person, the annotators were instructed to pick one and stick to that. Lastly, the annotators were provided with the peripheral annotation instructions found in Table 2.

While this identification process did include anaphora resolution of singular pronouns - such as resolving 'him' to 'Bran' - the annotators were instructed to ignore plural pronoun references. Plural pronoun resolution remains a difficult topic in the creation of social networks, as family members may sometimes be mentioned individually, and sometimes their family as a whole. Identifying group membership, and modelling that in the social network structure is not covered by any of the tools we include in our analysis or the related work referenced in Section 2 and therefore left to future work.

\begin{tabular}{l|l}
\hline Guideline & Example \\
\hline Ignore generic pronouns & $\begin{array}{l}\text { "Everyone knows; you } \\
\text { don't mess with me!" }\end{array}$ \\
\hline Ignore exclamations & "For Christ's sake!" \\
\hline $\begin{array}{l}\text { Ignore generic } \\
\text { noun phrases }\end{array}$ & $\begin{array}{l}\text { "Bilbo didn't know what } \\
\text { to tell the wizard." }\end{array}$ \\
\hline $\begin{array}{l}\text { Include non-human } \\
\text { named characters }\end{array}$ & $\begin{array}{l}\text { "His name is Buckbeak, } \\
\text { he's a hippogriph." }\end{array}$ \\
\hline
\end{tabular}

Table 2. Annotation Instructions

\section{NAMED ENTITY RECOGNITION EXPERIMENTS AND RESULTS}

We evaluate the performance of four different named entity recognition systems on the annotated novels: BookNLP (Bamman et al., 2014), Stanford NER(Finkel et al., 2005), Illinois Tagger (Ratinov and Roth, 2009) and IXA-Pipe-NERC (Agerri and Rigau, 2016). The BookNLP pipeline uses the 2014-01-04 release of Stanford NER tagger (Finkel et al., 2005) internally with the 7-class ontonotes model. As there have been several releases, and we focus on entities of type Person, we also evaluate the 2017-06-09 Stanford NER 4-class CoNLL model.

The results of the different Named Entity Recognition systems are presented in Table 3 


\footnotetext{
10 https: / / www.clips.uantwerpen.be/conl12002/ner/bin/conlleval.txt Last retrieved: 30 October 2017
}

for the classic novels, and Table 4 for the modern novels. All results are computed using the evaluation script used in the CoNLL 2002 and 2003 NER campaigns using the phrase-based evaluation setup. ${ }^{10}$ The systems are evaluated according to micro-averaged precision, recall and $F_{1}$ measure. Precision is the percentage of named entities found by the system that were correct. Recall is the percentage of named entities present in the text that are retrieved by the system. The $F_{1}$ measure is the harmonic mean of the precision and recall scores. In a phrase-based evaluation setup, the system only scores a point if the complete entity is correctly identified, thus if in a named entity consisting of multiple tokens only two out of three tokens are correctly identified, the system does not obtain any points.

The BookNLP and IXA-Pipe-NERC systems require that part of speech tagging is performed prior to named entity recognition, we use the modules included in the respective systems for this. For Stanford NER and Illinois NE Tagger plain text is offered to the NER systems.

As the standard deviations on the bottom rows of Tables 3 and 4 indicate, the results on the different books vary greatly. However, the different NER systems generally do perform similarly on the same novels, indicating that difficulties in recognising named entities in particular books is a characteristic of the novels rather than the systems. An exception is Brave New World on which BookNLP performs quite well, but the others underperform. Upon inspection, we find that the annotated chapter of this book contains only 5 different characters among which "The Director" which occurs 19 times. This entity is consistently missed by the systems resulting in a high penalty. Furthermore, the 'Mr.' in 'Mr. Foster' (occurring 31 times) is often not recognised as in some NE models titles are excluded. A token-based evaluation of Illinois NE Tagger on this novel for example yields a $\mathrm{F}_{1}$ score of 51.91. The same issue is at hand with Dr. Jekyll and Mr. Hyde and Dracula. Although the main NER module in BookNLP is driven by Stanford NER, we suspect that additional domain adaptations in this package account for this performance difference.

When comparing the $F_{1}$ scores of the $1^{\text {st }}$ person novels to the $3^{\text {rd }}$ person novels in Tables 3 and 4 , we find that the $1^{\text {st }}$ person novels perform significantly worse than their $3^{\text {rd }}$ person counterparts, at $p<.01$. These findings are in line with the findings of Elson et al. (2010).

In Section 6, we delve further into particular difficulties that fiction presents named entity recognition with and showcase solutions that do not require retraining the entity models.

As the BookNLP pipeline in the majority of the cases outperforms the other systems and includes coreference resolution and character clustering, we further utilise this system to create our networks. The results of the BookNLP pipeline including the coreference and clustering are presented in Table A4. One of the main differences in that table is that if popular entities are not recognised by the system they are penalised heavier because the coreferent mentions are also not recognised and linked to the correct entities. This results in scores that are generally somewhat lower, but the task that is measured is also more complex.

\section{NETWORK ANALYSIS}

In this section, we explain how the networks were created using the recognised named entities (Subsection 5.1), followed by an explanation of network analysis measures that we applied to compare the networks (Subsection 5.2). We discuss the results of the analysis (Subsection 5.3), as well as present an exploration of the network of one novel in particular to illustrate how a visualisation of a network can highlight particular characteristics of the interactions in the selected novel (Subsection 5.4). 


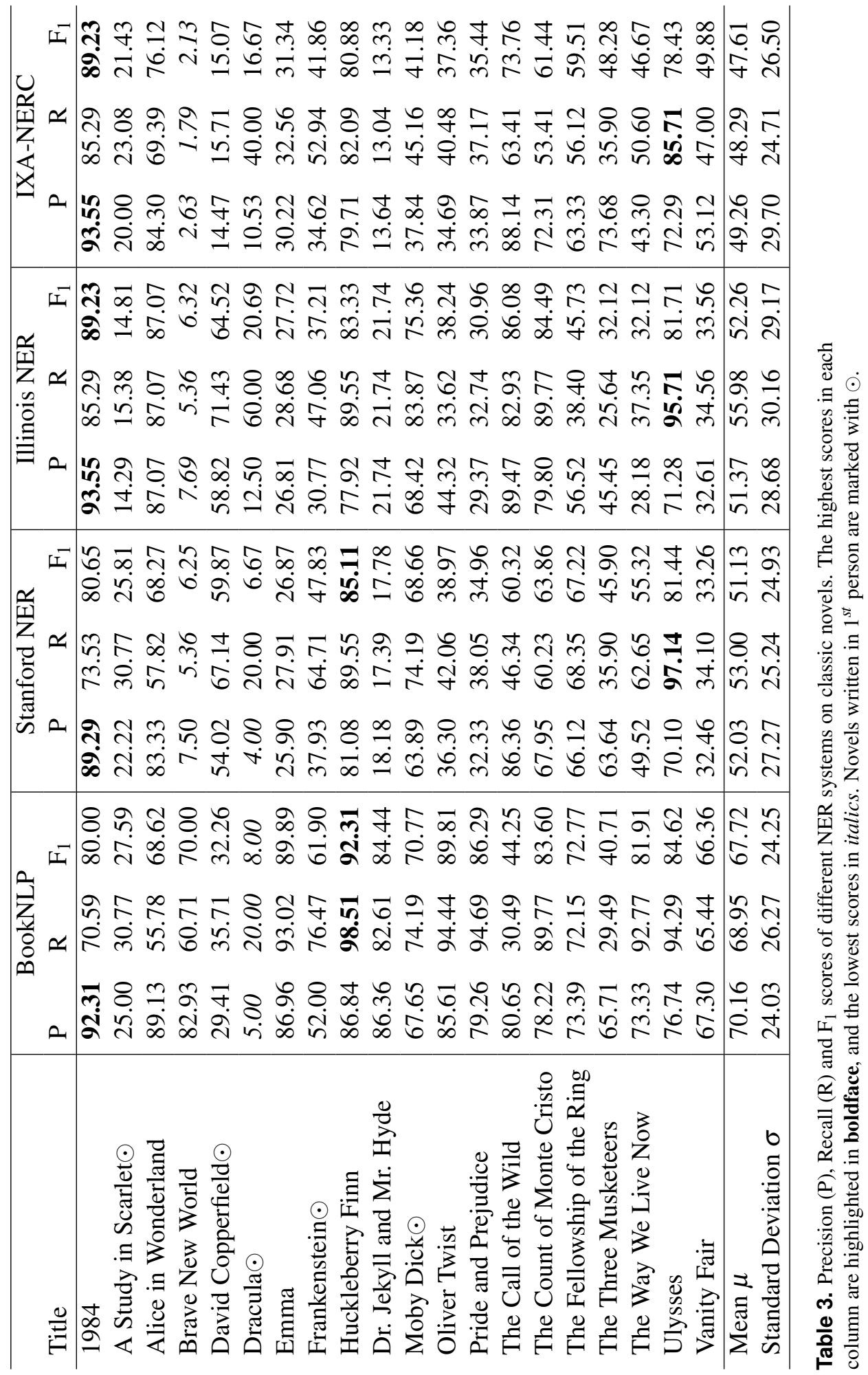




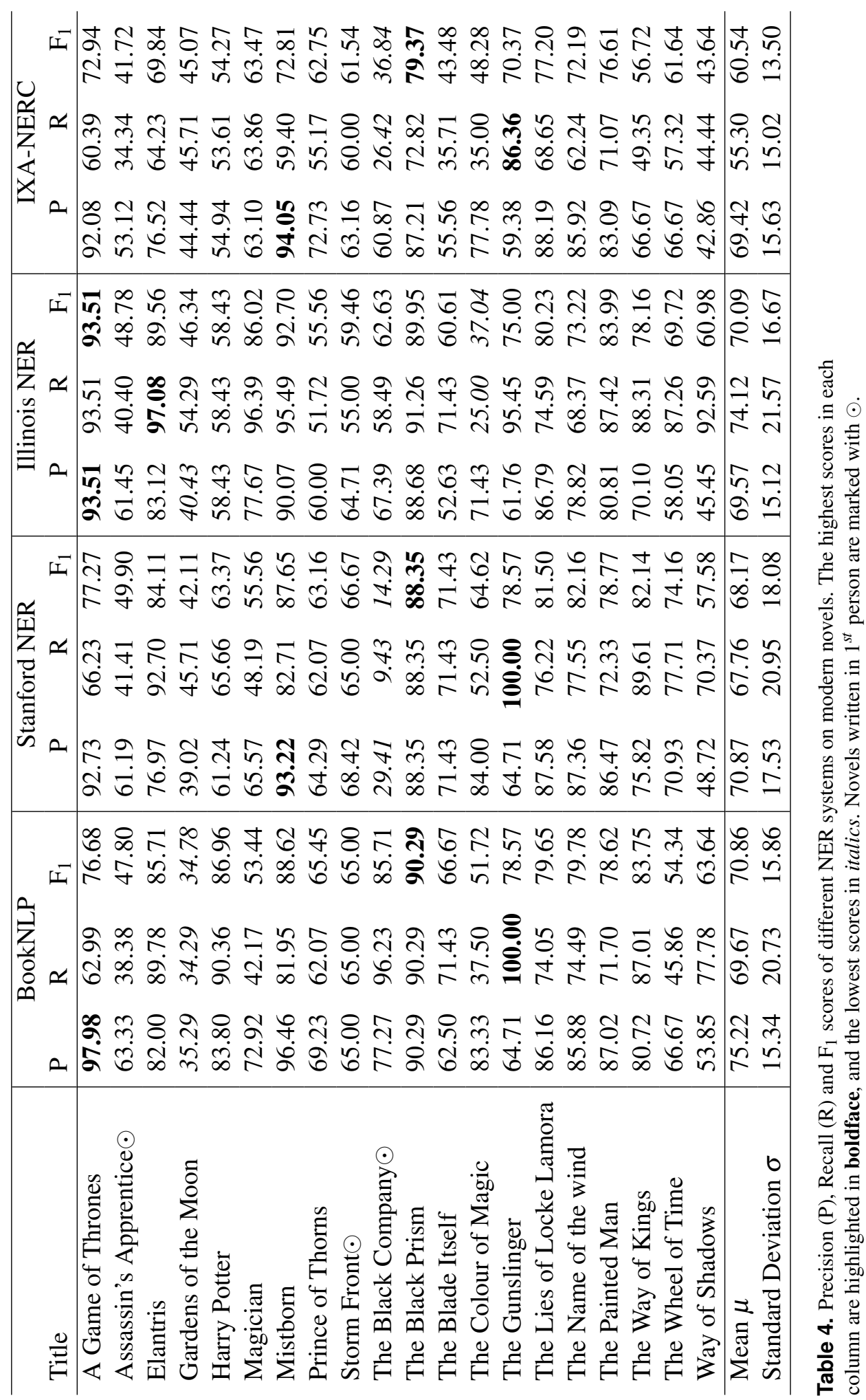




\subsection{Network Construction}

As explained in Section 2, we opt for the co-occurrence rather than the conversational method for finding the edges of our networks. The body of text that is used to define a co-occurrence differs per approach. Whereas Fernandez et al. (2015) define such a relation if characters are mentioned in the same sentence, Ardanuy and Sporleder (2014) use a paragraph for the same definition. We consider the delineation of what constitutes a paragraph to be too vague for the purpose of this study. While paragraphs are arguably better at conveying who interacts with whom, simply because of their increased length, it also brings forth an extra complexity in terms of their definition. Traditionally, paragraphs would be separated from another by means of a newline followed by an indented first line of the next paragraph. While this format holds for a part of our collection, it is not uniform. Other paragraph formats simply add vertical white space, or depend solely on the content (Bringhurst, 2004). Especially because the text files in our approach originate from different online sources - each with their own accepted format we decided that the added ambiguity should be avoided. For this study, we therefore define that a co-occurrence relationship between two characters exists if they are mentioned in the same sentence. For a co-occurrence of more than two characters, we follow Elson et al. (2010). That is, a multi-way co-occurrence between four characters is broken down into six bilateral co-occurrences.

For the construction of each social network, the co-occurrences are translated to nodes for characters and edges for relationships between the characters. We thus create a static, undirected and weighted graph. For the weight of each edge, we follow Ardanuy and Sporleder (2014). That is, each edge is assigned a weight depending on the number of interactions between two characters. For the construction of the network, we used NetworkX ${ }^{11}$ and Gephi ${ }^{12}$ to visualise the networks.

To ground the network analysis to be presented below, we gathered some overall statistics of the network creation process shown in Table A3 on page 24. As mentioned in Subsection 3.3, if the annotator decided that a character was definitely present, but unable to assert which character, the occurrence was marked as default. The fraction of defaults represents what portion of all identified characters was marked with default. The fraction of unidentified characters represents the percentage of characters that were not retrieved by the system, but had to be added by the annotators. Next, we present some overall statistics such as sentence length, the average number of persons in a sentence, and the average fraction of sentences that mention a person. Lastly, we kept track of the total number of annotated sentences, the total number of unique characters and character mentions. The only difference that could be identified between classes is the average sentence length, which was significant at $p<.01$. The sentences in classic books are significantly longer than in modern novels, suggesting that there is indeed some difference in writing style. However, other than that, none of the other measures differ significantly. This is useful information, as it helps support that the novels used in either class are comparable, despite their age-gap.

\subsection{Network Features}

We analyse the following eight network features:

1. Average degree is the mean degree of all the nodes in the network. The degree of a node is defined as the number of other nodes the node is connected to. If the degree of a node is 0 , the node is connected to no other nodes. The degree of a node in a social network is thus is measure of its social 'activity' (Wasserman and Faust, 1994). A high value -

\footnotetext{
${ }^{11}$ https: //networkx.github.io/ - v1.11

12 https://gephi.org/ - v0.9.1
} 
e.g. in Ulysses - indicates that the characters interact with many different other characters. Contrarily, a low value - e.g. in 1984 - indicates that the characters only interact with a small number of other characters.

2. Average Weighted Degree is fairly similar to the average degree, but especially in the sense of social networks, a distinction must be made. It differs in the sense that the weighted degree takes into account the weight of each of the connecting edges. Whereas a character in our social network could have a high degree - indicating a high level of social activity - if the weights of all those connected edges are relatively small, this suggests only superficial contact. Conversely, while the degree of a character could be low - e.g. the character is only connected to two other characters - the two edges could have very large weights, indicating a deep social connection between the characters. Newman (2006) underlines the importance of this distinction in his work on scientific collaborations. To continue the examples of Ulysses and 1984; while their average degrees are vastly different (with Ulysses being the highest of its class and 1984 the lowest), their average weighted degrees are comparable.

3. Average Path Length is the mean of all the possible shortest paths between each node in the network; also known as the geodesic distance. If there is no path connecting two nodes, this distance is infinite and the two nodes are part of different graph components (see item 7, Connected Components on the next page). The shortest path between two nodes can be found by using Dijkstra's algorithm (Dijkstra, 1959). The path length is typically an indication of how efficiently information is relayed through the network. A network with a low path length would indicate that the people in the network can reach each other through a relatively small number of steps.

4. Network Diameter is the longest possible distance between two nodes in the network. It is in essence the longest, shortest path that can be found between any two nodes in the network, and is indicative of the linear size of the network (Wasserman and Faust, 1994).

5. Graph density is the fraction of edges compared to the total number of possible edges. It thus indicates how complete the network is, where completeness would constitute all nodes being directly connected by an edge. This is often used in social network analysis to represent how closely the participants of the network are connected (Scott, 2012).

6. Modularity is used to represent community structure. The modularity of a network is "...the number of edges falling within groups minus the expected number in an equivalent network with edges placed at random" (Newman, 2006). Newman shows modularity can be used as an optimisation metric to approximate the number of community structures found in the network. To identify the community structures, we used the Louvain algorithm (Blondel et al., 2008). The identification of community structures in graph is useful, because the nodes in the same community are more likely to have other properties in common (Danon et al., 2005). It would therefore be interesting to see if differences can be observed between the prevalence of communities between the classic and modern novels.

7. Connected components are the number of distinct graph compartments. That is, a graph component is a subgraph in which any two vertices are connected to each other by paths, and which is connected to no additional vertices in the supergraph. In other words, it is not possible to traverse from one component to another. In most social communities, one 'giant component' can typically be identified, which contains the majority of all vertices (Kumar et al., 2010). A higher number of connected components would indicate a higher number of isolated communities. This is different from modularity in the sense that components are more strict. If only a single edge goes out from a subgraph to the 
supergraph, it is no longer considered a separate component. Modularity attempts to identify those communities that are basically 'almost' separate components.

8. Average clustering coefficient is the mean of all clustering coefficients. The clustering coefficient of a node can perhaps best be described as 'all-my-neighbours-know-eachother'. Social networks with a high clustering coefficient (and low average path length) may exhibit small world ${ }^{13}$ properties (Watts and Strogatz, 1998). The small world phenomenon was originally described by Stanley Milgram in his perennial work on social networks (Travers and Milgram, 1967).

\subsection{Results of Network Analysis}

To answer our second research question, we compared the network features presented in Subsection 5.2 for the social networks of the two different sets of novels. Table A5 on page 26 shows the results. The most striking feature of these results is the wide variance across social networks on all these network measures for both the classic and the modern novels. The size of these network ranges from just 10 nodes to networks more than 50 times as large. The network size alone can also explain at least a large part of the differences in graph density, diameter, and average path length, but also average degree and clustering coefficient show wide variation.

While we can observe large variation overall, there is no clear difference between the two classes, i.e. between classic and modern novels. None of the evaluated network features differ significantly between these classes. Graph density is the feature that comes closest to being significant $(p=0.09)$, with our classic novels on average exhibiting denser networks than the modern ones.

In order to better interpret these values, and in order to find out whether this variance in network features is by itself a characteristic property of social networks exposed in novels, or whether this is true for social networks in general, we need a point for comparison. For that purpose, we compare our network results to metrics that have been reported for other social network in the literature. Table 5 shows ten such networks for comparison, including three small networks on karate club members, football players, and email users (Telesford et al., 2011), three medium-sized networks of mathematicians, a larger group of email users, and actors (Boccaletti et al., 2006), and four large networks of online platforms (Mislove et al., 2007).

We can see that social networks reported elsewhere exhibit a wide variation as well, showing (unsurprisingly) an even much wider range for the network size, with the reported online social networks reaching millions of nodes. Our networks from novels are on the lower end of the size range, with the smallest ones being smaller than the smallest network of our comparison set (Karate). This directly explains why the path lengths are also on the lower end of the range, but with a considerable overlap. With respect to the average degree, our novel networks are covered by the range given by these comparison networks, with even the outliers of our dataset being less extreme than the most extreme cases of the comparison networks. The same holds for the clustering coefficient, except for the outlier for a very small network with a clustering coefficient of 0 (Alice in Wonderland). In summary, we can say that social networks from novels appear to be no different than social networks in general in showing a high variation in basically all network features across different networks. While networks differ much individually, there is no significant fundamental difference between classic and modern novels.

\subsection{Network Exploration}

In addition to the formal analysis above, we show here a more informal exploration of one of the networks in order to give a more intuitive explanation of our results. For that purpose, we selected

${ }^{13}$ https://en.wikipedia.org/wiki/Small-world_experiment 


\begin{tabular}{|c|c|c|c|c|c|}
\hline network & via & nodes & $\begin{array}{r}\text { average } \\
\text { degree }\end{array}$ & $\begin{array}{c}\text { clustering } \\
\text { coefficient }\end{array}$ & $\begin{array}{r}\text { average } \\
\text { path length }\end{array}$ \\
\hline Karate & Telesford et al. (2011) & $\mathbf{3 5}^{\dagger}$ & 4.46 & 0.55 & $2.41^{\dagger}$ \\
\hline Football & Telesford et al. (2011) & 115 & 10.66 & 0.40 & 2.51 \\
\hline E-mail & Telesford et al. (2011) & 1133 & 9.62 & 0.22 & 3.60 \\
\hline Math1999 & Boccaletti et al. (2006) & 58516 & 5.00 & 0.15 & $8.46^{\diamond}$ \\
\hline e-mail & Boccaletti et al. (2006) & 59812 & 2.88 & $\mathbf{0 . 0 3}{ }^{\dagger}$ & 4.95 \\
\hline Actors & Boccaletti et al. (2006) & 225226 & $61.00^{\diamond}$ & $0.79^{\diamond}$ & 3.65 \\
\hline YouTube & Mislove et al. (2007) & 1157827 & 1.81 & 0.14 & 5.10 \\
\hline Flickr & Mislove et al. (2007) & 1846198 & 1.76 & 0.31 & 5.67 \\
\hline Orkut & Mislove et al. (2007) & 3072441 & $\mathbf{1 . 5 0} 0^{\dagger}$ & 0.17 & 4.25 \\
\hline LiveJournal & Mislove et al. (2007) & $5284457^{\diamond}$ & 1.62 & 0.33 & 5.88 \\
\hline \multirow{3}{*}{ classic novels } & maximum & 522 & 15.77 & 0.81 & 3.33 \\
\hline & mean & 106 & 6.14 & 0.60 & 2.49 \\
\hline & minimum & 10 & 1.66 & 0.00 & 1.53 \\
\hline \multirow{3}{*}{ modern novels } & maximum & 314 & 10.50 & 0.75 & 4.06 \\
\hline & mean & 99 & 5.50 & 0.56 & 2.68 \\
\hline & minimum & 27 & 3.00 & 0.42 & 2.22 \\
\hline
\end{tabular}

Table 5. Comparison to other social networks. The highest scores in each column are highlighted with a $\diamond$ and the lowest scores with a $\uparrow$ for the comparison networks.

\footnotetext{
${ }^{14}$ MetaCPAN is a search engine for Perl code and documentation: https://metacpan. org/source/ BRIANL/Lingua-EN-Nickname-1.14/nicknames . txt Last Retrieved: 30 October 2017
} 


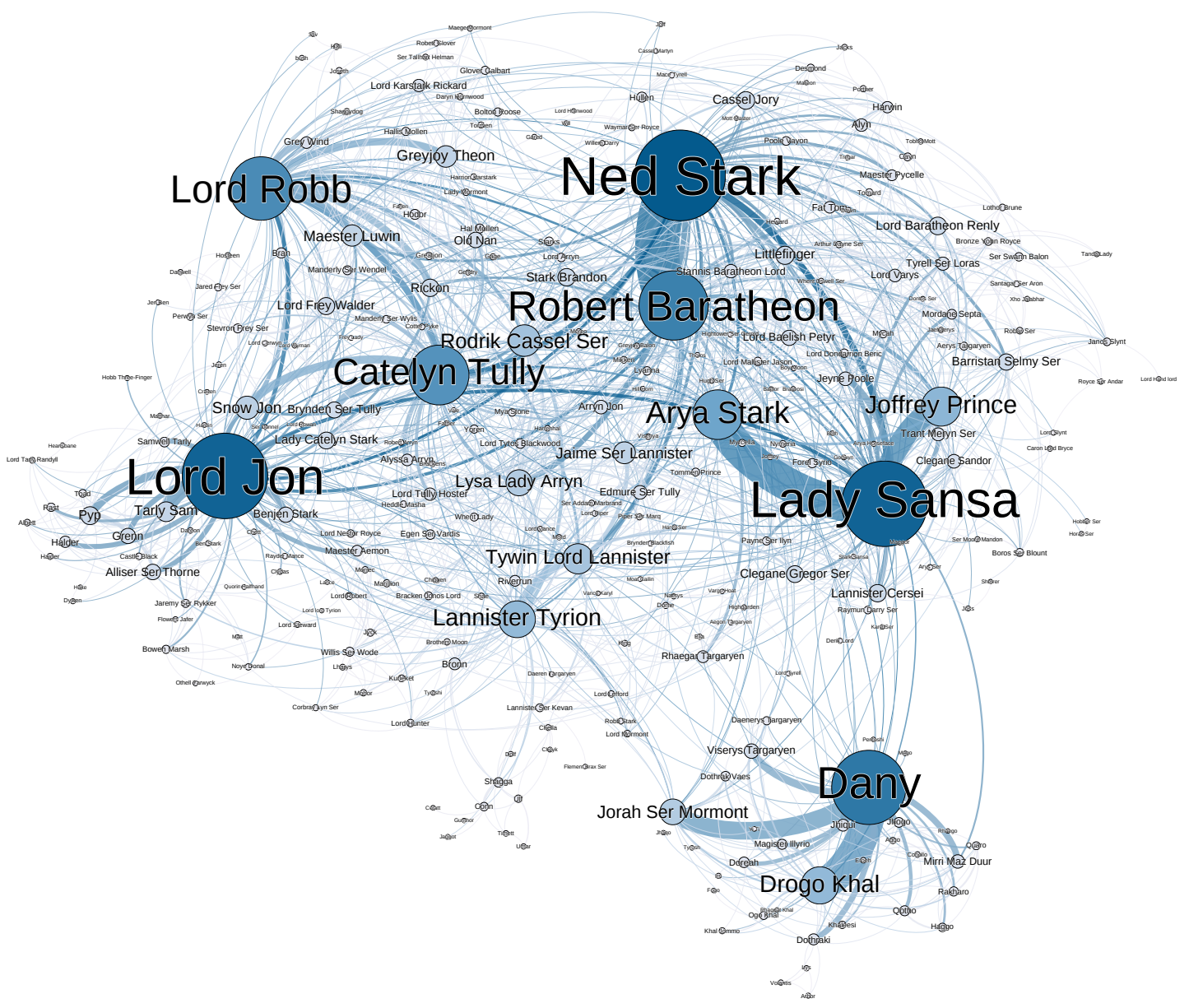

Figure 1. Social network of G.R.R. Martin's A Game of Thrones

is desirable to collapse those characters. Especially in A Game of Thrones, the mentions of Dany and Daenerys Targaryen occur in entirely different contexts. Whereas references to Dany occur in an environment that is largely friendly towards her; her formal name of Daenerys Targaryen is mostly used by her enemies (in her absence). Rather than simply collapsing the two characters as one, it might be useful to be able to retain that distinction. This is a design choice that will depend on the type of research question one wants to answer by analysing the social networks.

\section{DISCUSSION AND PERFORMANCE BOOSTING OPTIONS}

In analysing the output of the different NER systems, we found that some types of characters were particularly difficult to recognise. Firstly, we found a number of unidentified names that are so called word names (i.e. terms that also occur in dictionaries, for example to denote nouns such as Grace or Rebel). We suspected that this might hinder the named entity recognition, which is why we collected all such names in our corpus in Table A1 on page 22, and highlighted such word names with a $\dagger$. This table shows that approximately $50 \%$ of all unidentified names in our entire corpus consist at least partially of a word name, which underpins that this issue is potentially widely spread. In order to verify this, we replaced all potentially problematic names in the source material by generic English names. We made sure not to add names that were already assigned to other characters in the novel, and we ensured that these names were not also regular nouns. An example of these changed character names can be found in Table 6, which 


\begin{tabular}{l|l}
\hline Original & Adjusted \\
\hline Blue & Richard \\
Croaker & Thomas \\
Curly & Daniel \\
Dancing & Edward \\
Mercy & Charles \\
One-Eye & Timothy \\
Silent & James \\
Walleye & William \\
\hline
\end{tabular}

Table 6. Unidentified names in The Black Company replaced by generic English names.

shows all names affected for The Black Company.

Secondly, we noticed that persons with special characters in their names can prove difficult to retrieve. For example, names such as d'Artagnan in The Three Musketeers or Shai'Tan in The Wheel of Time were hard to recognise for the systems. To test this, we replaced all names in our corpus such as d'Artagnan or Shai'Tan with Dartagnan and Shaitan. By applying these transformations to our corpus, we found that the performances could be improved, uncovering some of the issues that plague named entity recognition. As can be observed in Figure 2, not all of the novels were affected by these transformations. Out of the 40 novels used in this study, we were able to improve the performance for 14. While the issue of the apostrophed affix was not as recurrent in our corpus as the real-word names, its impact on performance is troublesome nonetheless. Clearly, two novels are more affected by these transformations than the others, namely: The Black Company and the The Three Musketeers. To further sketch these issues, we delve a bit deeper into these two specific novels.

These name transformations show that the real-word names and names with special characters were indeed problematic and put forth a problem for future studies to tackle. As illustrated by Figure 2, the aforementioned issues are also present in the classic novels typically used by related works (such as The Three Musketeers). This begs the question of the scope of these problems. To the best of our knowledge, similar works have not identified this issue to affect their performances, but we have shown that with a relatively simple workaround, the performance can be drastically improved. It would thus be interesting to evaluate how much these studies suffer from the same issue. Lastly, as manually replacing names is clearly far from ideal, we would like to encourage future work to find a more robust approach to resolve this issue.

\section{The Black Company}

This fantasy novel describes the dealings of an elite mercenary unit - The Black Company - and its members, all of which go by code names such as the ones in Table 6 . With a preliminary $F_{1}$ score of 06.85 (see Table A4), The Black Company did not do very well. We found this book had the highest percentage of unidentified characters of our collection. Out of the 14 characters found by our annotators, only 5 were identified by the pipeline. Interestingly enough, 8 out of the 9 unidentified characters in this novel have names that correspond to regular nouns. By applying our name transformation alone, the $F_{1}$ score rose from 06.85 to the highest in our collection to 90.

\section{The Three Musketeers}

This classic piece recounts the adventures of a young man named d'Artagnan, after he leaves home to join the Musketeers of the Guard. With an $F_{1}$ score of 13.91 (see Table A4), The 


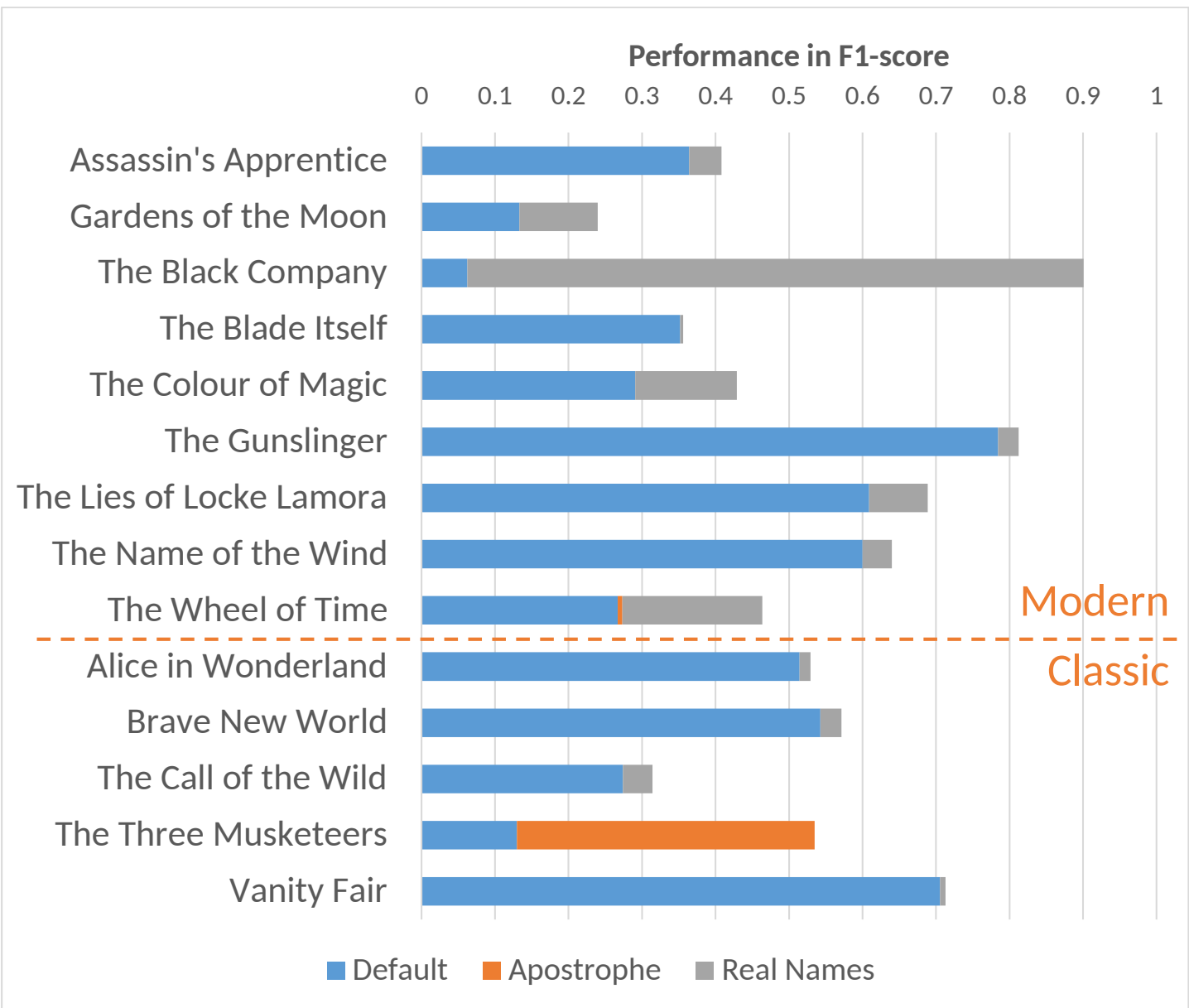

Figure 2. Effect of transformations on all affected classic and modern novels in $F_{1}$ score in using the BookNLP pipeline (includes co-reference resolution)

Three Musketeers performs the second worst of our corpus, and the worst in its class. By simply replacing names such as d'Artagnan with Dartagnan the $F_{1}$ score rose from 13.91 to 53, suggesting that the apostrophed name was indeed the main issue. To visualise this, we have included figures of both The Three Musketeer networks - before and after the fix - in Figures 3 and 4. As can be observed in Figure 3, the main character of the novel is hardly represented in this network, which is not indicative of the actual story. The importance of resolving the issue of apostrophed named is made clear in Figure 4, where the main character is properly represented.

\section{CONCLUSION \& FUTURE WORK}

In this study, we set out to close a gap in the literature when it comes to the evaluation of named entity recognition for the creation of social networks from fiction literature. In our exploration of related work, we found no other studies that attempt to compare networks from classic and modern fiction. To fill this gap, we attempted to answer the following two research questions:

- To what extent are off-the-shelf named entity recognition tools suitable for identifying fictional characters in novels?

- Which differences or similarities can be discovered between social networks extracted for different novels? 


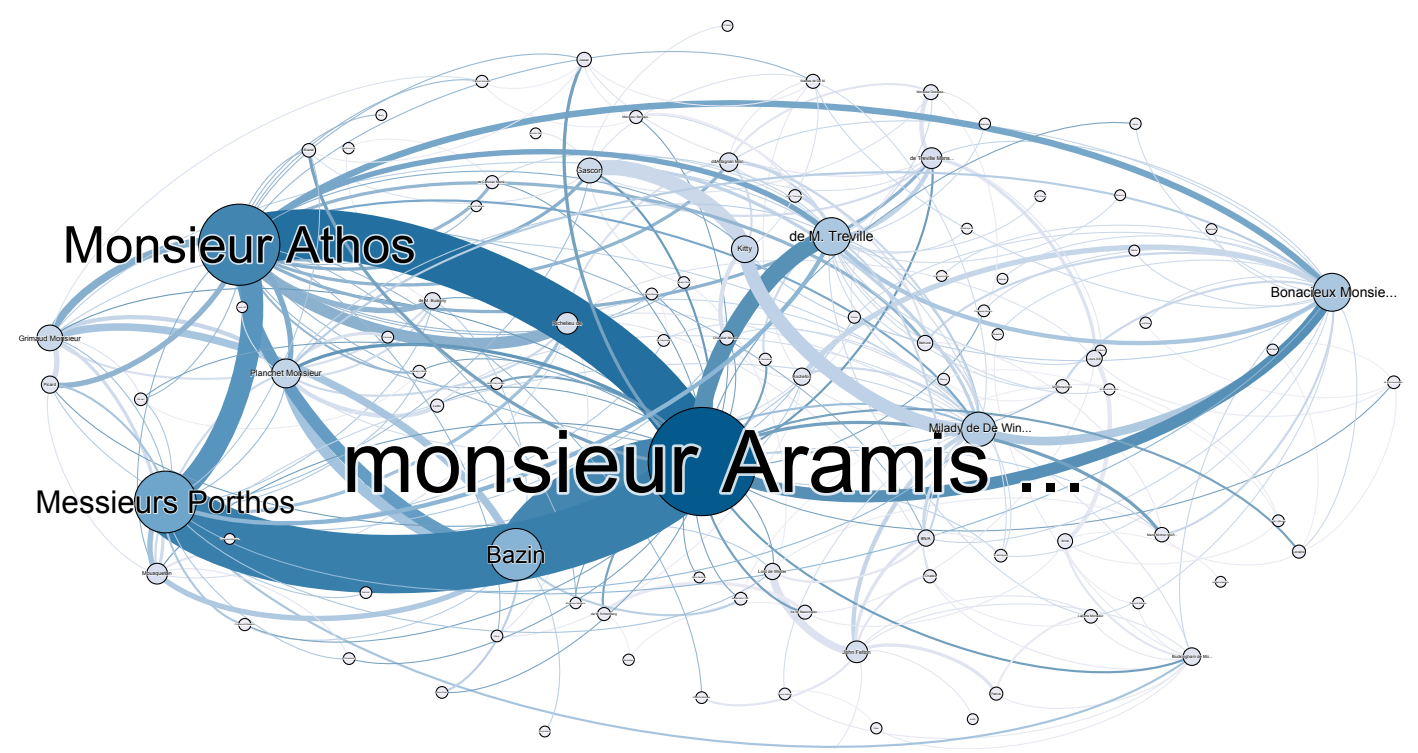

Figure 3. Social network of The Three Musketeers without adjustment for apostrophed names.

To answer our primary research question, we evaluated four state-of-the-art named entity recognition systems on 20 classic and 20 modern science fiction/fantasy novels. In our study, we found no significant difference in performance of the named entity recognisers on classic novels and modern novels. We did find that novels written in $3^{r d}$ person perspective perform significantly better than those written in $1^{\text {st }}$ person, which is in line with findings in related studies. In addition, we observed a large amount of variance within each class, even despite our limitation for the modern novels to the fantasy/science fiction genre. We also identified some recurring problems that hindered named entity recognition. We delved deeper into two such problematic novels, and find two main issues that overarch both classes. Firstly, we found that word names such as Mercy are more difficult to identify to the systems. We showed that replacing problematic word names by generic placeholders can increase performance on affected novels. Secondly, we found that apostrophed names such as d'Artagnan also prove difficult to automatically identify. With fairly simple methods that capture some cultural background knowledge, we circumvented the above two issues to drastically increase the performance of the used pipeline. To the best of our knowledge, none of the related studies discussed in Section 2 acknowledge the presence of these issues. We would thus like to encourage future work to evaluate the impact of these two issues on existing studies, and call to develop a more robust approach to tackle them in future studies.

To answer our secondary research question, we created social networks for each of the novels in our collection and calculated several networks features with which we compared the two classes. As with the named entity recognition experiments, no major differences were found between the classic and modern novels. Again, we found that the distribution of network measures within a class was subject to high variance, which holds for our collection of both classic and modern novels. We therefore recommend that future work focuses on determining particular characteristics that can influence these analyses first and then perform a comparative analysis between subsets to see if this similarity between classes holds when the variance is reduced. Future studies could therefore attempt to compare classic and modern novels in the same genre or narration type (e.g. first-person vs third-person perspective). Lastly, different types of networks that for example collapse characters that occur under different names (cf. Dany and Daenerys) as well as dealing with plural pronouns and group membership (e.g. characters 


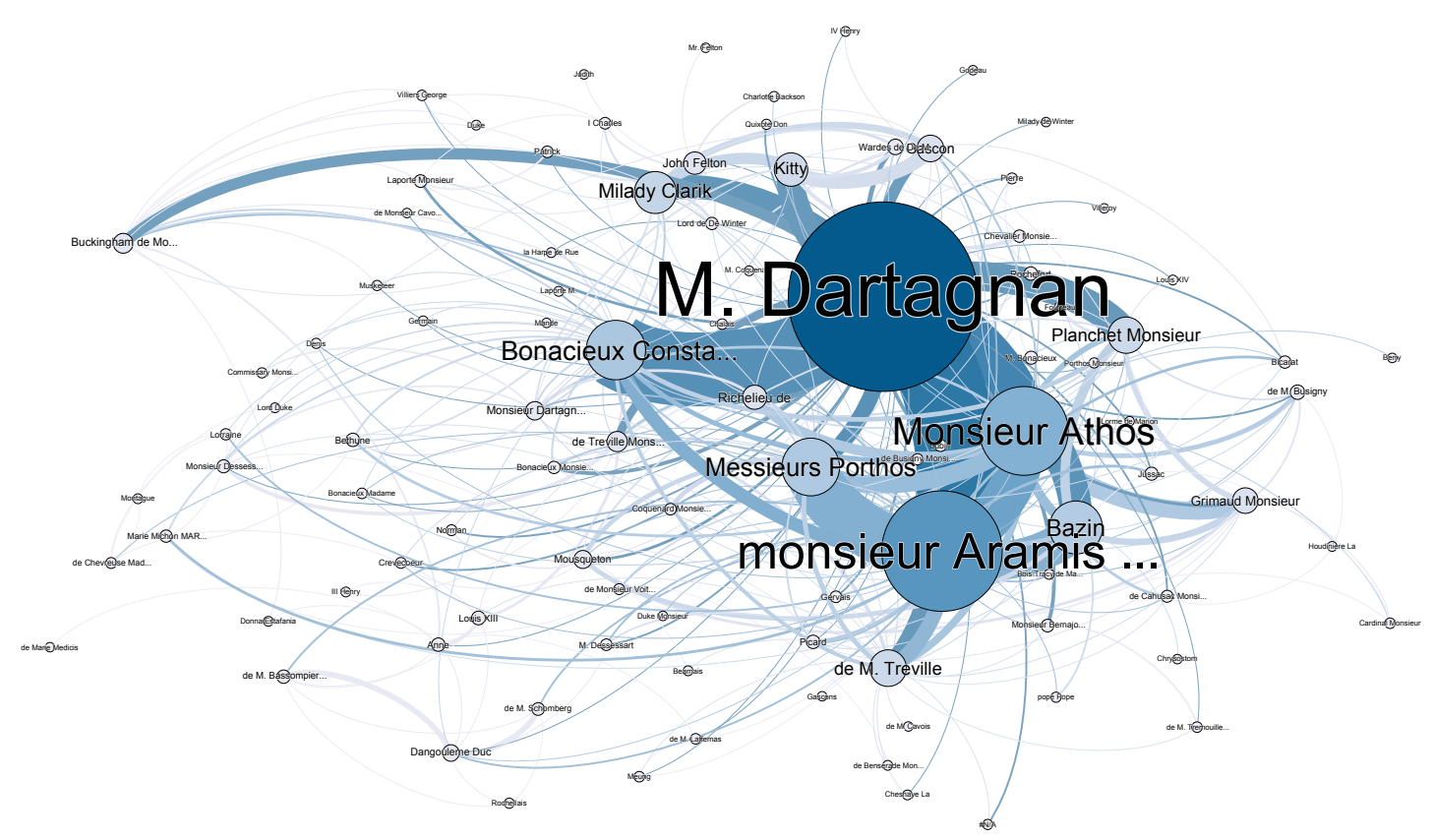

Figure 4. Social network of The Three Musketeers with adjustment for apostrophed names.

sometimes mentioned individually and sometimes as part of a group) are currently unsolved problems for language technology and knowledge representation. These issues point to a strong need for more culturally-aware artificial intelligence.

\section{REFERENCES}

Agarwal, A., Corvalan, A., Jensen, J., and Rambow, O. (2012). Social network analysis of alice in wonderland. In Proceedings of the NAACL-HLT 2012 Workshop on computational linguistics for literature, pages 88-96.

Agarwal, A., Kotalwar, A., and Rambow, O. (2013). Automatic extraction of social networks from literary text: A case study on alice in wonderland. In Sixth International Joint Conference on Natural Language Processing (IJNLP 2013), pages 1202-1208.

Agerri, R. and Rigau, G. (2016). Robust multilingual named entity recognition with shallow semi-supervised features. Artificial Intelligence, 238:63-82.

Akimushkin, C., Amancio, D. R., and Oliveira Jr, O. N. (2017). Text authorship identified using the dynamics of word co-occurrence networks. PloS one, 12(1):e0170527.

Amancio, D. R. (2015). Probing the topological properties of complex networks modeling short written texts. PloS one, 10(2):e0118394.

Ardanuy, M. C. and Sporleder, C. (2014). Structure-based clustering of novels. In Proceedings of the EACL Workshop on Computational Linguistics for Literature, pages 31-39.

Bamman, D., Underwood, T., and Smith, N. A. (2014). A bayesian mixed effects model of literary character. In Proceedings of The 52nd Annual Meeting of the Association for Computational Linguistics (ACL 2014), pages 370-379.

Biber, D. and Finegan, E. (1989). Drift and the evolution of english style: A history of three genres. Language, 65(3):487 - 517.

Blondel, V. D., Guillaume, J.-L., Lambiotte, R., and Lefebvre, E. (2008). Fast unfolding of communities in large networks. Journal of statistical mechanics: theory and experiment, 2008(10):P10008. 
Boccaletti, S., Latora, V., Moreno, Y., Chavez, M., and Hwang, D.-U. (2006). Complex networks: Structure and dynamics. Physics reports, 424(4-5):175-308.

Bringhurst, R. (2004). The elements of typographic style. Hartley \& Marks Vancouver, British Columbia.

Chambers, N. and Jurafsky, D. (2008). Unsupervised learning of narrative event chains. In The 46th Annual Meeting of the Association for Computational Linguistics (ACL 2008), volume 94305, pages 789-797. Association for Computational Linguistics.

Danon, L., Diaz-Guilera, A., Duch, J., and Arenas, A. (2005). Comparing community structure identification. Journal of Statistical Mechanics: Theory and Experiment, 2005(09):P09008.

de Does, J., Depuydt, K., van Dalen-Oskam, K., and Marx, M. (2017). Namescape: Named entity recognition from a literary perspective. In Odijk, J. and van Hessen, A., editors, CLARIN in the Low Countries, page 361?370. Ubiquity Press. License: CC-BY 4.0.

Dijkstra, E. W. (1959). A note on two problems in connexion with graphs. Numerische mathematik, 1(1):269-271.

Elson, D. K., Dames, N., and McKeown, K. R. (2010). Extracting social networks from literary fiction. In Proceedings of the 48th annual meeting of the association for computational linguistics, pages 138-147. Association for Computational Linguistics.

Elson, D. K. and McKeown, K. (2010). Automatic attribution of quoted speech in literary narrative. In The Twenty-Fourth AAAI Conference on Artificial Intelligence (AAAI-2010). Association for the Advancement of Artificial Intelligence.

Fernandez, M., Peterson, M., and Ulmer, B. (2015). Extracting social network from literature to predict antagonist and protagonist. Technical report, Stanford University.

Finkel, J. R., Grenager, T., and Manning, C. (2005). Incorporating non-local information into information extraction systems by gibbs sampling. In Proceedings of the 43rd annual meeting on association for computational linguistics, pages 363-370. Association for Computational Linguistics.

He, H., Barbosa, D., and Kondrak, G. (2013). Identification of speakers in novels. In Proceedings of the 51st Annual Meeting of the Association for Computational Linguistics (ACL 2013), pages $1312-1320$.

Kumar, R., Novak, J., and Tomkins, A. (2010). Structure and evolution of online social networks. In Link mining: models, algorithms, and applications, pages 337-357. Springer.

Lee, J. and Yeung, C. Y. (2012). Extracting networks of people and places from literary texts. In Proceedings of the 26th Pacific Asia Conference on Language, Information, and Computation (PACLIC 2012), pages 209-218.

Mac Carron, P. and Kenna, R. (2012). Universal properties of mythological networks. EPL (Europhysics Letters), 99(2):28002.

Mislove, A., Marcon, M., Gummadi, K. P., Druschel, P., and Bhattacharjee, B. (2007). Measurement and analysis of online social networks. In Proceedings of the 7th ACM SIGCOMM conference on Internet measurement, pages 29-42. ACM.

Moretti, F. (2013). Distant reading. Verso Books.

Newman, M. E. (2006). Modularity and community structure in networks. Proceedings of the national academy of sciences, 103(23):8577-8582.

Ratinov, L. and Roth, D. (2009). Design challenges and misconceptions in named entity recognition. In Proceedings of the Thirteenth Conference on Computational Natural Language Learning (CoNLL-2009), pages 147-155, Boulder, Colorado. Association for Computational Linguistics.

Sainte-Beuve, C. A. (1910). What is a classic? In Eliot, C. W., editor, Literary and Philosophical Essays: French, German and Italian, volume 32 of The Harvard classics. P. F. Collier \& Son Corporation. 
Scott, J. (2012). Social network analysis. Sage.

Telesford, Q. K., Joyce, K. E., Hayasaka, S., Burdette, J. H., and Laurienti, P. J. (2011). The ubiquity of small-world networks. Brain connectivity, 1(5):367-375.

Travers, J. and Milgram, S. (1967). The small world problem. Phychology Today, 1:61-67.

Vala, H., Jurgens, D., Piper, A., and Ruths, D. (2015). Mr. bennet, his coachman, and the archbishop walk into a bar but only one of them gets recognized: On the difficulty of detecting characters in literary texts. In EMNLP, pages 769-774.

van Dalen-Oskam, K., de Does, J., Marx, M., Sijaranamual, I., Depuydt, K., Verheij, B., and Geirnaert, V. (2014). Named entity recognition and resolution for literary studies. Computational Linguistics in the Netherlands Journal, 4:121-136.

Wasserman, S. and Faust, K. (1994). Social network analysis: Methods and applications, volume 8. Cambridge university press.

Watts, D. J. and Strogatz, S. H. (1998). Collective dynamics of small-world-networks. nature, 393(6684):440-442. 
APPENDIX: ADDITIONAL STATISTICS

\begin{tabular}{|c|c|c|c|c|}
\hline \multicolumn{3}{|c|}{ Classic } & \multicolumn{2}{|c|}{ Modern } \\
\hline $\begin{array}{l}\text { Ada } \\
\text { Algy } \\
\text { Alice } \\
\text { Anna Boleyne } \\
\text { Aprahamian } \\
\text { Belisarius } \\
\text { Best-Ingram } \\
\text { Cain } \\
\text { Caroline } \\
\text { Catherine } \\
\text { Cato } \\
\text { Cervantes } \\
\text { Christine } \\
\text { Chuck Loyola } \\
\text { Cleopatra } \\
\text { Connolly Norman } \\
\text { Curly } \\
\text { Dante }^{\dagger} \\
\text { Dave } \\
\text { Dives } \\
\text { Dodo } \\
\text { Dr. Floss } \\
\text { Duck }^{\dagger} \\
\text { Edgar Atheling }^{\dagger} \\
\text { Elmo } \\
\text { Farmer Mitchell } \\
\text { Father Joseph }^{\dagger} \\
\text { Fury }^{\dagger} \\
\text { Ginny }_{\text {Henry VIII }}\end{array}$ & 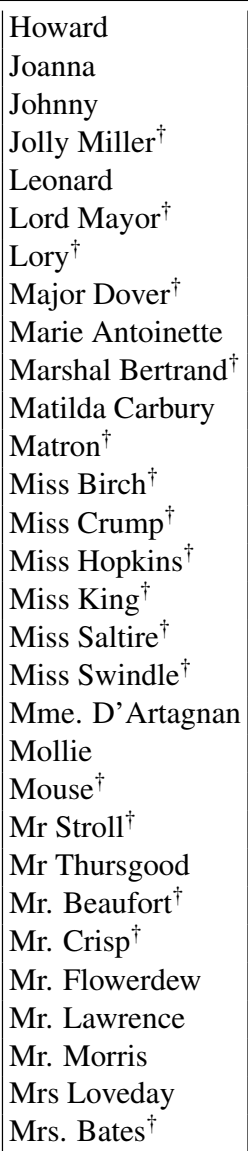 & \begin{tabular}{|l|} 
Mrs. Billington \\
Mrs. Birch $^{\dagger}$ \\
Mrs. Crisp $^{\dagger}$ \\
Mrs. Effington Stubbs \\
Mrs. Thingummy \\
Murray \\
Nathan Swain $^{\dagger}$ \\
Peter Teazle $^{\dagger}$ \\
Policar Morrel $^{\dagger}$ \\
President West $^{\dagger}$ \\
Queequeg \\
Rip Van Winkle \\
Royce $^{\dagger}$ \\
Sawbones \\
Semiramis \\
Shep \\
Sir Carbury \\
Skrimshander \\
Stamford \\
Stigand \\
Sudeley \\
Swubble \\
The Director \\
Tommy Barnes \\
Unwin \\
Ursula \\
Victor $^{\dagger}$ \\
Vilkins \\
Von Bischoff $^{\dagger}$ \\
Ysabel $^{\dagger}$
\end{tabular} & $\mid$\begin{tabular}{l|} 
Archmage of Ymitury \\
August $^{\dagger}$ \\
Bil Baker $^{\dagger}$ \\
Blue $^{\dagger}$ \\
Brine Cutter $^{\dagger}$ \\
Bug $^{\dagger}$ \\
Chyurda \\
Cotillion \\
Croaker $^{\dagger}$ \\
Curly $^{\dagger}$ \\
Dadda \\
Dancing \\
Domi \\
Dow \\
Elam Dowtry \\
Elao \\
Fredor \\
Gart \\
Harold \\
Harvey \\
Howard \\
Ien \\
Ilgrand Lender \\
Ishar \\
Ishi \\
Jim McGuffin \\
Kerible the Enchanter $^{\dagger}$ \\
Lilly \\
$\dagger$
\end{tabular} & $\begin{array}{l}\text { Manie } \\
\text { Meena } \\
\text { Mercy } \\
\text { Mrs. Potter }^{\dagger} \\
\text { Old Cob }^{\dagger} \\
\text { One-Eye }^{\dagger} \\
\text { Pappa Doc }^{\dagger} \\
\text { Patience }^{\dagger} \\
\text { Plowman }^{\dagger} \\
\text { Poul } \\
\text { Rand }^{\dagger} \\
\text { Shalash }^{\dagger} \\
\text { Shrewd } \\
\text { Silent } \\
\text { Sirius } \\
\text { Talenel }^{\dagger} \\
\text { Talenelat } \\
\text { Ted } \\
\text { The Empress } \\
\text { Themos Tresting } \\
\text { Theron } \\
\text { Threetrees } \\
\text { Toffston } \\
\text { Verus } \\
\text { Walleye } \\
\text { Weasel } \\
\text { Willum } \\
\text { Wit Congar }^{\dagger}\end{array}$ \\
\hline \multicolumn{3}{|c|}{39 out of 90 characters: $43 \%$} & \multicolumn{2}{|c|}{30 out of 56 characters: $54 \%$} \\
\hline
\end{tabular}

Table A1. Characters that were not identified by the system, supplied by the annotators. Characters whose names (partly) consist of a real word - such as 'Curly' or 'Mercy' - are marked with a $\uparrow$. Checked against http://dictionary.com. 


\begin{tabular}{|c|c|c|c|}
\hline \multicolumn{4}{|c|}{ Classic } \\
\hline Title & Author & (Year) & E-book No. / ISBN \\
\hline 1984 & George Orwell & (1949) & 9780451518651 \\
\hline A Study in Scarlet & Conan Doyle & $(1886)$ & 244 \\
\hline Alice in Wonderland & Lewis Carroll & $(1884)$ & 19033 \\
\hline Brave New World & Aldous Huxley & $(1865)$ & 9780965185196 \\
\hline David Copperfield & Charles Dickins & $(1931)$ & 766 \\
\hline Dracula & Bram Stoker & (1850) & 345 \\
\hline Emma & Jane Austen & (1897) & 158 \\
\hline Frankenstein & Mary Shelley & $(1815)$ & 84 \\
\hline Huckleberry Finn & Mark Twain & $(1818)$ & 76 \\
\hline Jekyll and Hyde & Robert Stevenson & $(1851)$ & 42 \\
\hline Moby Dick & Herman Melville & $(1838)$ & 2701 \\
\hline Oliver Twist & Charles Dickins & (1813) & 730 \\
\hline Pride and Prejudice & Jane Austen & $(1886)$ & 1342 \\
\hline The Call of the Wild & Jack London & (1903) & 215 \\
\hline The Count of Monte Cristo & Alexandre Dumas & $(1844)$ & 1184 \\
\hline The Fellowship of the Ring & J. R. R. Tolkien & (1954) & 9780547952017 \\
\hline The Three Musketeers & Alexandre Dumas & (1844) & 1257 \\
\hline The Way We Live Now & Anthony Trollope & $(1875)$ & 5231 \\
\hline Ulysses & James Joyce & (1922) & 4300 \\
\hline Vanity Fair & William Thackeray & $(1847)$ & 599 \\
\hline \multicolumn{4}{|c|}{ Modern } \\
\hline Title & Author & (Year) & E-book No. / ISBN \\
\hline A Game of Thrones & G.R.R. Martin & (1996) & 9780307292094 \\
\hline Assassin's Apprentice & Robin Hobb & (1995) & 9781400114344 \\
\hline Elantris & Brandon Sanderson & (2005) & 9780765383105 \\
\hline Gardens of the Moon & Steven Erikson & (1999) & 9788498003178 \\
\hline Harry Potter & J.K. Rowling & (1998) & 9781781103685 \\
\hline Magician & Raymond Feist & (1982) & 9780007466863 \\
\hline Mistborn & Brandon Sanderson & (2006) & 9788374805537 \\
\hline Prince of Thorns & Mark Lawrence & (2011) & 9786067192681 \\
\hline Storm Front & Jim Butcher & $(2000)$ & 9781101128657 \\
\hline The Black Company & Glen Cook & (1984) & 9782841720743 \\
\hline The Black Prism & Brent Weeks & (2010) & 9782352945260 \\
\hline The Blade Itself & Joe Abercrombie & (2006) & 9781478935797 \\
\hline The Colour of Magic & Terry Pratchett & $(1983)$ & 9788374690973 \\
\hline The Gunslinger & Steven King & (1982) & 9781501143519 \\
\hline The Lies of Locke Lamora & Scott Lynch & (2006) & 9780575079755 \\
\hline The Name of the Wind & Patrick Rothfuss & (2007) & 9782352949152 \\
\hline The Painted Man & Peter Brett & (2008) & 9780007518616 \\
\hline The Way of Kings & Brandon Sanderson & (2010) & 9780765326355 \\
\hline The Wheel of Time & Robert Jordan & $(1990)$ & 9781857230765 \\
\hline Way of Shadows & Brent Weeks & $(2008)$ & 9781607513513 \\
\hline
\end{tabular}

Table A2. Classic and modern novels included in this study. The short E-book numbers are the catalog entry of novels obtained from Gutenberg. Novels obtained through online purchase are denoted by the longer ISBNs. 


\begin{tabular}{|c|c|c|c|c|c|c|c|c|}
\hline \multicolumn{9}{|c|}{ Classic } \\
\hline Title & 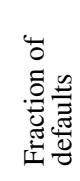 & 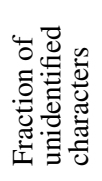 & 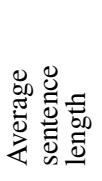 & 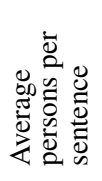 & 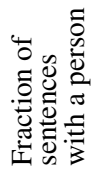 & 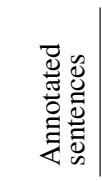 & 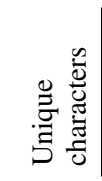 & 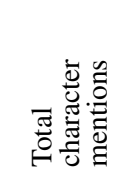 \\
\hline 1984 & 0.55 & $\mathbf{0 . 0 0}^{\dagger}$ & 18.01 & 1.17 & 0.32 & 316 & 29 & 2162 \\
\hline A Study in Scarlet & 0.83 & 0.50 & 18.99 & 1.17 & 0.18 & 193 & 34 & 837 \\
\hline Alice in Wonderland & 0.26 & $0.56^{\triangleright}$ & 20.99 & 1.23 & 0.79 & 316 & 17 & 656 \\
\hline Brave New World & 0.35 & 0.17 & 15.87 & 1.06 & 0.25 & 299 & 51 & 1809 \\
\hline David Copperfield & 0.61 & $\mathbf{0 . 0 0}^{\dagger}$ & 22.79 & 1.08 & 0.49 & 261 & 157 & 9922 \\
\hline Dracula & $0.93^{\diamond}$ & $\mathbf{0 . 0 0}^{\dagger}$ & 21.96 & $1.00^{\dagger}$ & $0.066^{\dagger}$ & 233 & 72 & 3369 \\
\hline Emma & 0.43 & 0.10 & 22.38 & 1.38 & 0.81 & 224 & 78 & 6946 \\
\hline Frankenstein & 0.86 & 0.22 & 25.80 & 1.19 & 0.17 & 300 & 29 & 658 \\
\hline Huckleberry Finn & 0.59 & 0.14 & 23.46 & 1.20 & 0.40 & 215 & 82 & 1749 \\
\hline Jekyll and Hyde & 0.67 & 0.29 & 26.19 & 1.17 & 0.34 & $\mathbf{1 2 0}^{\dagger}$ & $\mathbf{1 3}^{\dagger}$ & $\mathbf{5 2 3}^{\dagger}$ \\
\hline Moby Dick & 0.88 & 0.38 & 25.24 & 1.10 & 0.10 & 442 & 135 & 2454 \\
\hline Oliver Twist & 0.36 & 0.33 & 21.64 & 1.23 & 0.68 & 303 & 69 & 4495 \\
\hline Pride and Prejudice & 0.46 & 0.10 & 24.13 & 1.48 & 0.79 & 257 & 62 & 5104 \\
\hline The Call of the Wild & 0.49 & 0.50 & 21.67 & 1.31 & 0.61 & 192 & 28 & 731 \\
\hline The Count of Monte Cristo & 0.47 & 0.25 & 21.91 & 1.35 & 0.79 & 197 & 250 & 13562 \\
\hline The Lord of the Rings & 0.47 & 0.48 & 16.30 & 1.20 & 0.46 & $\mathbf{7 6 9}$ & 134 & 5268 \\
\hline The Three Musketeers & 0.60 & 0.36 & 19.19 & 1.13 & 0.49 & 265 & 115 & 4842 \\
\hline The Way We Live Now & 0.57 & 0.46 & 18.93 & 1.14 & 0.47 & 341 & 147 & 13993 \\
\hline Ulysses & 0.57 & 0.33 & $\mathbf{1 3 . 3 5}^{\dagger}$ & 1.15 & 0.41 & 303 & $651^{\diamond}$ & 8510 \\
\hline Vanity Fair & 0.24 & 0.44 & $27.27^{\diamond}$ & $1.54^{\diamond}$ & $1.05^{\diamond}$ & 256 & 359 & 11503 \\
\hline Mean $\mu$ & 0.56 & 0.28 & 21.30 & 1.21 & 0.48 & 290.10 & 125.60 & 4954.65 \\
\hline Standard Deviation $\sigma$ & 0.20 & 0.18 & 3.67 & 0.14 & 0.27 & 131.89 & 150.20 & 4403.32 \\
\hline \multicolumn{9}{|c|}{ Modern } \\
\hline A Game of Thrones & 0.29 & $\mathbf{0 . 0 0}^{\dagger}$ & 14.53 & 1.30 & $0.82^{\diamond}$ & 283 & $322^{\diamond}$ & $158399^{\diamond}$ \\
\hline Assassin's Apprentice & 0.71 & 0.29 & 14.94 & 1.18 & 0.38 & 460 & 66 & 2857 \\
\hline Elantris & 0.32 & 0.27 & 14.24 & 1.10 & 0.60 & 367 & $\mathbf{1 4}^{\dagger}$ & $226^{\dagger}$ \\
\hline Gardens of the Moon & 0.75 & 0.44 & 12.20 & $1.03^{\dagger}$ & 0.25 & 304 & 111 & 4479 \\
\hline Harry Potter & 0.32 & 0.33 & 15.55 & 1.33 & 0.74 & 338 & 84 & 5114 \\
\hline Magician & 0.49 & 0.17 & 14.78 & 1.16 & 0.45 & 310 & 115 & 4976 \\
\hline Mistborn & 0.34 & 0.22 & 12.90 & 1.19 & 0.68 & 297 & 104 & 11672 \\
\hline Prince of Thorns & 0.54 & $\mathbf{0 . 0 0}^{\dagger}$ & 12.33 & 1.14 & 0.38 & 107 & 79 & 2282 \\
\hline Storm Front & 0.77 & $\mathbf{0 . 0 0}{ }^{\dagger}$ & 14.02 & 1.05 & 0.18 & 211 & 43 & 2368 \\
\hline The Black Company & 0.56 & $0.64^{\circ}$ & $9.73^{\dagger}$ & 1.07 & 0.26 & 305 & 42 & 1908 \\
\hline The Black Prism & 0.50 & 0.14 & 13.19 & 1.04 & 0.40 & 380 & 88 & 10890 \\
\hline The Blade Itself & 0.66 & 0.29 & 12.55 & 1.14 & 0.24 & 103 & 107 & 6769 \\
\hline The Colour of Magic & 0.55 & 0.50 & 14.21 & 1.12 & 0.42 & 139 & 34 & 1454 \\
\hline The Gunslinger & $0.78^{\diamond}$ & 0.25 & 13.43 & 1.11 & $\mathbf{0 . 1 7 ^ { \dagger }}$ & 230 & 35 & 1159 \\
\hline The Lies of Locke Lamora & $0.21^{\dagger}$ & 0.09 & $16.90^{\diamond}$ & $1.38^{\diamond}$ & 0.77 & 305 & 105 & 6477 \\
\hline The Name of the Wind & 0.45 & 0.10 & 12.98 & 1.14 & 0.45 & 310 & 137 & 6405 \\
\hline The Painted Man & 0.30 & 0.28 & 14.67 & 1.29 & 0.70 & 301 & 137 & 9048 \\
\hline The Way of Kings & 0.31 & 0.29 & 12.20 & 1.10 & 0.36 & 316 & 221 & 14696 \\
\hline The Wheel of Time & 0.40 & 0.21 & 14.96 & 1.31 & 0.59 & $499^{\diamond}$ & 188 & 9426 \\
\hline Way of Shadows & 0.32 & 0.13 & 13.53 & 1.32 & 0.56 & $\mathbf{8 8}^{\dagger}$ & 160 & 8721 \\
\hline Mean $\mu$ & 0.48 & 0.23 & 13.69 & 1.17 & 0.47 & 282.65 & 109.60 & 6338.30 \\
\hline Standard Deviation $\sigma$ & 0.18 & 0.17 & 1.54 & 0.11 & 0.20 & 110.52 & 72.98 & 4535.60 \\
\hline$\mu_{\text {classic }}-\mu_{\text {modern }}$ & 0.08 & 0.05 & 7.61 & 0.04 & 0.01 & 7.45 & 16.00 & -1383.65 \\
\hline Pooled $\sigma$ & 0.20 & 0.17 & 2.46 & 0.24 & 0.25 & 125 & 119 & 4473 \\
\hline$p$-value & 0.21 & 0.39 & ¿ 0.01 & 0.73 & 0.74 & 0.85 & 0.68 & 0.35 \\
\hline Significant & No & No & Yes & No & No & No & No & No \\
\hline
\end{tabular}

Table A3. Overall statistics for classic and modern novels in our corpus. The highest scores in each column are highlighted with a $\diamond$, and the lowest scores with a $\dagger$. The highest and lowest performing books for each class, in terms of $F_{1}$ score found in Tables 3 and 4, are marked with a grey fill. 


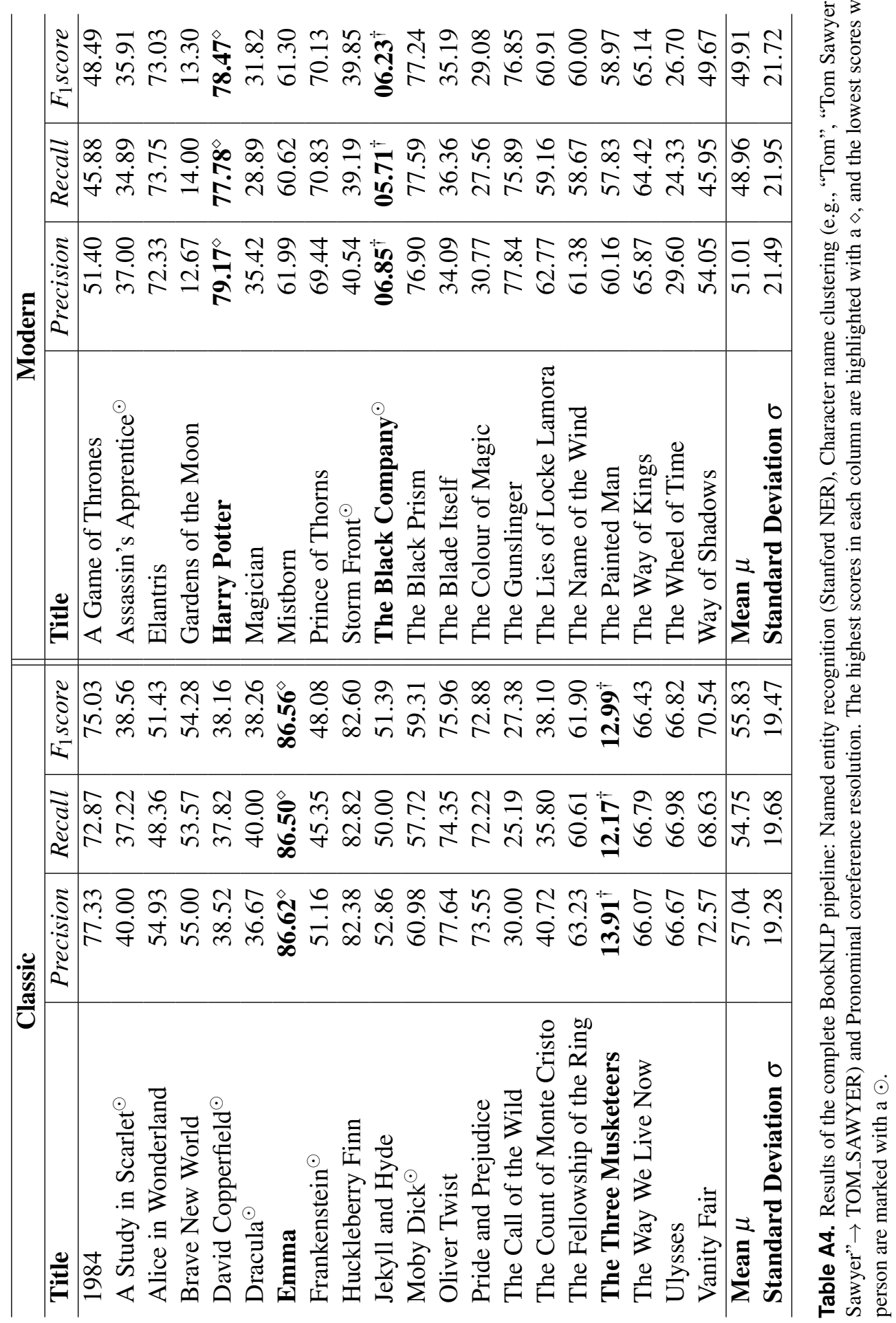




\begin{tabular}{|c|c|c|c|c|c|c|c|c|c|c|}
\hline \multicolumn{11}{|c|}{ Classic } \\
\hline Title & $\begin{array}{l}\tilde{\theta} \\
\bar{z} \\
z\end{array}$ & 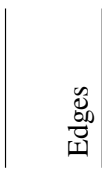 & 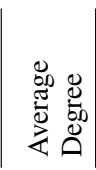 & 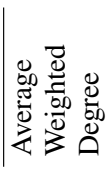 & 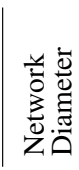 & 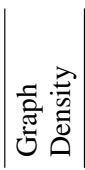 & 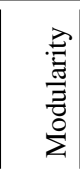 & 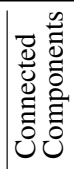 & 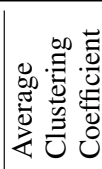 & 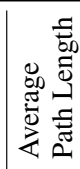 \\
\hline 1984 & 26 & 43 & 3.30 & 16.84 & 4 & 0.13 & 0.23 & 3 & 0.5 & 2.06 \\
\hline A Study in Scarlet & 24 & 41 & 3.41 & 7.25 & 5 & 0.14 & 0.42 & 2 & 0.63 & 2.37 \\
\hline Alice in Wonderland & 12 & $\mathbf{1 0}^{\dagger}$ & $1.66^{\dagger}$ & $\mathbf{3 . 8 3}^{\dagger}$ & 3 & 0.15 & 0.15 & 2 & $\mathbf{0}^{\dagger}$ & 1.93 \\
\hline Brave New World & 39 & 65 & 3.33 & 9.79 & 6 & 0.09 & 0.34 & 2 & 0.68 & 2.53 \\
\hline David Copperfield & 142 & 499 & 7.03 & 23.11 & 6 & 0.05 & 0.49 & 2 & 0.57 & 2.69 \\
\hline Dracula & 55 & 124 & 4.51 & 18.29 & 6 & 0.08 & $0.12^{\dagger}$ & 4 & 0.52 & 2.53 \\
\hline Emma & 72 & 403 & 11.19 & $\mathbf{5 7 . 5 3}$ & 4 & 0.16 & 0.14 & 1 & 0.67 & 2.16 \\
\hline Frankenstein & 20 & 38 & 3.80 & 10.60 & 5 & 0.20 & 0.51 & 2 & 0.75 & 2.41 \\
\hline Huckleberry Finn & 62 & 121 & 3.90 & 8.42 & 7 & 0.06 & $0.52^{\diamond}$ & 4 & 0.60 & 3.30 \\
\hline Jekyll and Hyde & $\mathbf{1 0}^{\dagger}$ & 21 & 4.20 & 14.60 & $2^{\dagger}$ & $0.47^{\diamond}$ & 0.12 & 1 & $0.81^{\diamond}$ & $1.533^{\dagger}$ \\
\hline Moby Dick & 90 & 169 & 3.76 & 7.38 & 8 & 0.04 & 0.44 & 8 & 0.59 & $\mathbf{3 . 3 3}^{\diamond}$ \\
\hline Oliver Twist & 62 & 191 & 6.16 & 22.32 & 4 & 0.10 & 0.32 & 2 & 0.75 & 2.26 \\
\hline Pride and Prejudice & 62 & 373 & 12.03 & 57.10 & 4 & 0.20 & 0.16 & 1 & 0.73 & 1.96 \\
\hline The Call of the Wild & 23 & 44 & 3.83 & 10.00 & 6 & 0.17 & 0.46 & 1 & 0.62 & 2.46 \\
\hline The Count of Monte Cristo & 228 & 799 & 7.01 & 24.05 & 7 & 0.03 & 0.40 & 3 & 0.56 & 2.88 \\
\hline The Fellowship of the Ring & 105 & 260 & 4.95 & 11.51 & 6 & 0.05 & 0.29 & 2 & 0.63 & 2.73 \\
\hline The Three Musketeers & 96 & 279 & 5.81 & 15.33 & 5 & 0.06 & 0.32 & 1 & 0.55 & 2.56 \\
\hline The Way We Live Now & 135 & 630 & 9.33 & 39.17 & 5 & 0.07 & 0.36 & 3 & 0.69 & 2.43 \\
\hline Ulysses & 522 & $4116^{\diamond}$ & $15.77^{\diamond}$ & 18.59 & $\mathbf{9}^{\diamond}$ & 0.03 & 0.45 & $10^{\diamond}$ & 0.60 & 3.02 \\
\hline Vanity Fair & 342 & 1349 & 7.89 & 22.73 & 7 & $\mathbf{0 . 0 2} \mathbf{2}^{\dagger}$ & 0.37 & 1 & 0.63 & 2.72 \\
\hline Mean $\mu$ & 106 & 479 & 6.14 & 20 & 5.45 & 0.12 & 0.33 & 2.75 & 0.60 & 2.49 \\
\hline Standard Deviation $\sigma$ & 126.94 & 916.66 & 3.56 & 14.99 & 1.70 & 0.10 & 0.14 & 2.39 & 0.17 & 0.44 \\
\hline \multicolumn{11}{|c|}{ Modern } \\
\hline A Game of Thrones & $314^{\diamond}$ & $1648^{\diamond}$ & $10.50^{\diamond}$ & 22.46 & 6 & 0.03 & 0.48 & 1 & 0.54 & 2.81 \\
\hline Assassin's Apprentice & 55 & 110 & 4.00 & 9.09 & 6 & 0.07 & 0.34 & 2 & 0.49 & 2.65 \\
\hline Elantris & 106 & 493 & 9.30 & $43.25^{\diamond}$ & 5 & 0.09 & 0.36 & 1 & 0.67 & $2.22^{\dagger}$ \\
\hline Gardens of the Moon & 88 & 257 & 5.84 & 10.84 & 8 & 0.07 & 0.42 & 1 & 0.48 & 2.93 \\
\hline Harry Potter & 67 & 198 & 5.9 & 19.37 & 5 & 0.09 & 0.15 & 1 & 0.68 & 2.23 \\
\hline Magician & 84 & 209 & 4.98 & 10.76 & 6 & 0.06 & 0.43 & 2 & 0.58 & 2.83 \\
\hline Mistborn & 89 & 255 & 5.73 & 33.89 & 6 & 0.07 & $\mathbf{0 . 0 4}$ & 3 & 0.62 & 2.37 \\
\hline Prince of Thorns & 59 & 111 & 3.76 & 6.98 & 6 & 0.07 & 0.37 & 2 & $\mathbf{0 . 4 2}{ }^{\dagger}$ & 2.83 \\
\hline Storm Front & 33 & 85 & 5.15 & 10.97 & $4^{\dagger}$ & $0.16^{\diamond}$ & 0.31 & 1 & 0.64 & 2.26 \\
\hline The Black Company & 30 & 45 & $\mathbf{3 . 0 0} \dagger^{\dagger}$ & $6.13^{\dagger}$ & 6 & 0.10 & 0.20 & 3 & 0.561 & 2.43 \\
\hline The Black Prism & 84 & 239 & 5.69 & 30.74 & 5 & 0.07 & 0.22 & 1 & $0.75^{\diamond}$ & 2.27 \\
\hline The Blade Itself & 96 & 259 & 5.40 & 14.23 & 5 & 0.06 & 0.51 & 3 & 0.51 & 2.65 \\
\hline The Colour of Magic & $27^{\dagger}$ & $\mathbf{4 3}^{\dagger}$ & 3.19 & 7.93 & 6 & 0.12 & 0.38 & 1 & 0.50 & 2.67 \\
\hline The Gunslinger & 31 & 69 & 4.45 & 8.52 & 7 & 0.15 & 0.41 & 1 & 0.43 & 2.87 \\
\hline The Lies of Locke Lamora & 101 & 261 & 5.17 & 22.24 & 5 & 0.05 & 0.18 & 4 & 0.64 & 2.46 \\
\hline The Name of the Wind & 109 & 197 & 3.62 & 8.99 & $\mathbf{9}^{\diamond}$ & 0.03 & $0.67^{\diamond}$ & 5 & 0.46 & $4.06^{\diamond}$ \\
\hline The Painted Man & 132 & 444 & 6.73 & 23.15 & 7 & 0.05 & 0.53 & 1 & 0.63 & 2.70 \\
\hline The Way of Kings & 172 & 448 & 5.21 & 20.79 & 6 & $\mathbf{0 . 0 3}{ }^{\dagger}$ & 0.57 & $\mathbf{9}^{\diamond}$ & 0.55 & 2.91 \\
\hline The Wheel of Time & 167 & 545 & 6.53 & 16.66 & 7 & 0.04 & 0.35 & 3 & 0.55 & 2.84 \\
\hline Way of Shadows & 145 & 441 & 6.08 & 22.14 & 6 & 0.04 & 0.46 & 4 & 0.61 & 2.71 \\
\hline Mean $\mu$ & 99 & \begin{tabular}{|l}
317 \\
$\mid$
\end{tabular} & 5.50 & 17 & 6.05 & 0.07 & 0.36 & 2.45 & 0.56 & 2.68 \\
\hline Standard Deviation $\sigma$ & 66.37 & 348.92 & 1.85 & 10.05 & 1.15 & 0.04 & 0.15 & 1.99 & 0.09 & 0.4 \\
\hline$\mu_{\text {classic }}-\mu_{\text {modern }}$ & 7 & 162 & 0.64 & 3 & $\mid-0.60$ & 0.05 & $\mid-0.03$ & 0.30 & 0.04 & $\mid-0.19$ \\
\hline Pooled $\sigma$ & 101 & 695 & 2.83 & 12.83 & 1.45 & 0.08 & 0.15 & 2.18 & 0.13 & 0.43 \\
\hline$p$-value & 0.83 & 0.47 & 0.49 & 0.55 & 0.20 & 0.09 & 0.42 & 0.67 & 0.37 & 0.17 \\
\hline Significant & No & No & No & No & No & No & No & No & No & No \\
\hline
\end{tabular}

Table A5. Social network measures for classic and modern novels. The highest scores in each column are highlighted with a $\diamond$, and the lowest scores with a $\dagger$. The highest and lowest performing books for each class, in terms of $F_{1}$ score found in Tables 3 and 4, are marked with a grey fill. 